Setting An online survey for all continental football federations.

Patients (or Participants) A total of 1690 professional and semi-professional soccer players and coaches completed the survey.

Interventions (or Assessment of Risk Factors) The questionnaire consisted of questions relating to the awareness level, implementation rate, and opinion of the FIFA $11+$ injury prevention program. Questions development was guided by several authors whose expertise is in sport medicine and injury prevention.

Main Outcome Measurements The primary outcomes were awareness level, implementation rate, and opinion of the effectiveness of the FIFA $11+$ injury prevention program in reducing injuries.

Results A total of 824 (48.8\%) professional and semi-professional soccer players and coaches reported awareness of the FIFA $11+$ injury prevention program and 680 (40.2\%) reported implementing the FIFA $11+$ injury prevention program in their current practice at some level. Participants who implemented the FIFA $11+$ injury prevention program reported a positive attitude towards program efficacy, with a score of $8.16 \pm 1.10$ out of 10 .

Conclusions The results suggest a relationship between the variables explored. Therefore, increasing awareness of the FIFA +11 injury prevention program may increase implementation rates around the world, which may lead to an increase in the reduction of soccer injury incidence.

\section{INJURY PREVENTION KNOWLEDGE, BELIEFS, AND PRACTICES AMONG WOMEN'S FOOTBALL TEAMS IN SOUTH AFRICA}

Ummukulthoum Bakare, Benita Olivier, Corlia Brandt, Lonwabo Godlwana. University of the Witwatersrand, Johannesburg, South Africa

\subsection{6/bjsports-2021-IOC.384}

Background Injury prevention, or the lack thereof, is influenced by a variety of elements in any team context. With the rising number of injuries in women's football and the scarcity of human resources in Sub-Saharan Africa, it's critical to investigate how standardized injury prevention measures may be implemented.

Objective The goal of the study was to assess injury prevention knowledge, beliefs, and practices among women's football teams in the University Sports South Africa (USSA) Football League in South Africa's Gauteng Province.

Design This research design is a cross-sectional survey of injury prevention knowledge, beliefs, and practices.

Setting Women's football teams in the USSA Football league in South Africa's Gauteng Province.

Patients (or Participants) All women's teams in the USSA Football League in Gauteng and their support staff (mostly head coaches, assistant coaches, team managers, and occasional medical personnel) were asked to participate.

Interventions (or Assessment of Risk Factors) Not Applicable Main Outcome Measurements Perceived knowledge, beliefs, and practices.

Results Out of 107 participants, 35.5\% $(n=38)$ reported they perceived that they had adequate knowledge about injury prevention. It was also shown that $75.7 \%(n=81)$ of the participants were engaged in football injury prevention programmes (IPP)s at the time of the research, with the following injury prevention practices being relevant: warmups (95.1\%), stretching (90.1\%), cool-downs (80.2\%) and jogging (81.4\%). Only $24.9 \%$ of the respondents indicated that they had heard about the FIFA 11+ IPP, while the majority $(83.2 \%)$ stated that they would be willing to adopt it if they were to gain access to detailed information on the programme.

Conclusions The majority of their football teammates thought their knowledge was insufficient. Most players and coaches believe that IPPs are important, which is an important first step towards developing and implementing injury prevention awareness programmes.

\section{SALIVARY SECRETORY IMMUNOGLOBULIN A IN MALE OLYMPIC FOOTBALL PLAYERS}

${ }^{1}$ Joao Brito, ${ }^{1,2}$ Figueiredo Pedro. ${ }^{1}$ Portugal Football School, Portuguese Football Federation, Oeiras, Portugal; ${ }^{2}$ Research Center in Sports Science, Health Sciences and Human Development, Maia, Portugal

\subsection{6/bjsports-2021-IOC.385}

Background Salivary secretory immunoglobulin A (sIgA) is a non-invasive biological marker that may be used to monitor an athlete's response to training and to identify athletes at risk for upper respiratory tract infection. However, its usefulness in elite athletes is unclear.

Objective To determine the variation of sIgA in male Olympic athletes during the preparation period and the Olympic football tournament.

Design Prospective observational study.

Setting Preparation period and the Olympic football tournament in Rio2016 (from July 18 to August 11, 2016).

Participants Twenty male elite football players (aged $22 \pm 2$ years) called up to the Olympic team.

Intervention Over 27 days, athletes had 19 training days, 2 traveling days, 2 days with friendly matches, and 4 days with official games.

Main Outcome Measurements sIgA was analysed using a real-time lateral flow device. Training and match internal loads were assessed using the session rating of perceived exertion (sRPE) method.

Results During the first 3 days of training, sRPE $(p<0.001)$, but not $\operatorname{sIgA}(\mathrm{p}>0.05)$, varied significantly; however, $\operatorname{sigA}$ had a moderate negative within-subjects correlation with sRPE $(\mathrm{r}=-0.39$; confidence interval 95\%: -0.62 to -0.09$)$. During the whole study period, daily sIgA ranged from $350 \pm 242$ to $517 \pm 238 \mu \mathrm{g} \cdot \mathrm{mL}^{-1}$ ( $\mathrm{p}>0.05$ ). The between-subjects coefficient of variation of sIgA ranged from $44.5 \%$ to $69.2 \%$, whereas its within-subjects coefficient of variation ranged from $17.3 \%$ to $74.7 \%$. Daily sRPE varied significantly throughout the whole study period $(\mathrm{p}<0.001)$. No upper respiratory tract infection was reported.

Conclusions sIgA showed a high between- and within-subjects variation in male elite football players, which may limit its usefulness as a valid biological marker for response to training and risk of upper respiratory tract infection. 